\begin{tabular}{|c|c|}
\hline Title & Frontier Molecular Orbital Based A nalysis of Solid-A dsorbate Interactions over Group 13 Metal Oxide Surfaces \\
\hline Author(s) & $\begin{array}{l}\text { Liu, Chong; Li, Y uxin; Takao, Motoshi; Toyao, Takashi; Maeno, Zen; Kamachi, Takashi; Hinuma, Y oyo; Takigawa, } \\
\text { Ichigaku; Shimizu, Ken-ichi }\end{array}$ \\
\hline Citation & $\begin{array}{l}\text { Journal of physical chemistry c, } 124(28), 15355-15365 \\
\text { https://doi.org/10.1021/acs.jpcc.0c04480 }\end{array}$ \\
\hline Issue Date & $2020-07-16$ \\
\hline Doc URL & http:/hdl.handle.net/2115/82235 \\
\hline Rights & $\begin{array}{l}\text { This document is the A ccepted Manuscript version of a Published Work that appeared in final form in The Journal of } \\
\text { Phy sical Chemistry C, copyright @ } @ \text { A merican Chemical Society after peer review and technical editing by the publisher. } \\
\text { To access the final edited and published work see https://pubs.acs.org/doi/10.1021/acs.jpcc.0c04480. }\end{array}$ \\
\hline Type & article (author version) \\
\hline File Information & main text for HUSCAP.pdf \\
\hline
\end{tabular}

Instructions for use 


\section{A Frontier Molecular Orbital Based Analysis of Solid-Adsorbate Interactions over Group 13 Metal Oxide Surfaces}

Chong Liu, ${ }^{1}$ Yuxin Li, ${ }^{1}$ Motoshi Takao, ${ }^{1}$ Takashi Toyao, ${ }^{*, 1,2}$ Zen Maeno, ${ }^{1}$ Takashi Kamachi, ${ }^{2,3}$ Yoyo Hinuma, ${ }^{4,5}$ Ichigaku Takigawa, ${ }^{6,7}$ and Ken-ichi Shimizu ${ }^{*, 1,2}$

${ }^{1}$ Institute for Catalysis, Hokkaido University, N-21, W-10, Sapporo, 001-0021, Japan

${ }^{2}$ Elements Strategy Initiative for Catalysts \& Batteries, Kyoto University, Katsura, Kyoto 6158520, Japan

${ }^{3}$ Department of Life, Environment and Applied Chemistry, Fukuoka Institute of Technology (FIT), 3-30-1 Wajiro-Higashi, Higashi-ku, Fukuoka 819-0295, Japan

${ }^{4}$ Center for Frontier Science, Chiba University, 1-33 Yayoicho, Inage, Chiba 263-8522, Japan

${ }^{5}$ Center for Materials Research by Information Integration, Research and Services Division of Materials Data and Integrated System, National Institute for Materials Science, 1-2-1 Sengen, Tsukuba, Ibaraki 305-0047, Japan

${ }^{6}$ RIKEN Center for Advanced Intelligence Project, 1-4-1 Nihonbashi, Chuo-ku, Tokyo 103-0027, Japan

${ }^{7}$ Institute for Chemical Reaction Design and Discovery (WPI-ICReDD), Hokkaido University, N21, W-10, Sapporo, Hokkaido 001-0021, Japan 


\section{ABSTRACT}

Adsorption is an essential process that takes place in heterogeneous catalysis. In the current study, solidadsorbate interactions occurring between a variety of small molecules and surfaces of group 13 metal oxides, including $\beta-\mathrm{Ga}_{2} \mathrm{O}_{3}(100), \beta-\mathrm{Ga}_{2} \mathrm{O}_{3}(001), \theta-\mathrm{Al}_{2} \mathrm{O}_{3}(100), \theta-\mathrm{Al}_{2} \mathrm{O}_{3}(001), \theta-\mathrm{Al}_{2} \mathrm{O}_{3}(010), \ln _{2} \mathrm{O}_{3}(110)$ and $\mathrm{In}_{2} \mathrm{O}_{3}(111)$, were investigated using DFT calculations and a machine learning (ML)-based statistical method. The adsorbates utilized for this purpose include $\mathrm{CO}, \mathrm{CO}_{2}, \mathrm{~N}_{2}, \mathrm{NH}_{3}, \mathrm{H}_{2} \mathrm{O}$, acetonitrile, acetone, acetamide, acetic acid, alkanes, alkenes, aromatic compounds, alcohols, and amines. The results show that the adsorption energies ( $E_{\text {ads }}$ ) of each metal oxide surface correlate linearly with the highest occupied molecular orbital (HOMO) energies of the adsorbates and not with energies of the lowest unoccupied molecular orbital (LUMO) of the small molecules. Moreover, in these systems, contributions to molecular adsorption are dominated by interactions between the HOMOs of the adsorbates and the surface conduction band of the metal oxides. Furthermore, surface energy was found to be an important parameter influencing $E_{\text {ads }}$ values of different metal oxides. Finally, the results of statistical analysis using a ML approach confirmed that adsorbate HOMOs and surface energy of metal oxides are the most influential factors governing molecular adsorption, and also demonstrated that dipole moments of adsorbates contribute to controlling to adsorption.

KEYWORDS: Frontier molecular orbital, group 13 metal oxides, DFT calculation, solid-adsorbate interactions, machine learning (ML), partial dependence plot (PDP) 


\section{INTRODUCTION}

Heterogeneous catalysis is an empirical science because of the complexities of surface interactions and reactions involved, which make the rationalization and prediction of catalyst performance a formidable challenge. ${ }^{1-3}$ As adsorption is a fundamental step in reactions taking place on surfaces, an especially important component of the discovery of novel heterogeneous catalysts and catalytic processes is an understanding of how reactants are coordinated to and activated on solid surfaces. However, despite their obvious importance to the field of heterogeneous catalysis, approaches to explore these issues in a systematic fashion and to develop methods for reliable predictions have not been fully developed.

Since the time of its introduction in the $1950 \mathrm{~s},{ }^{4}$ frontier molecular orbital (FMO) theory has been widely used to analyze and predict molecular interactions and reactivities of molecules. Numerous examples now exist, which show that reaction profiles of organic substances can be explained and even in some cases predicted by analyzing coefficients and energies of highest occupied molecular orbitals (HOMOs) and lowest unoccupied molecular orbitals (LUMOs). Although the utility of FMO theory in dealing with molecular interactions/reactions has been fully demonstrated, it has not yet been sufficiently shown that this theoretical method can be applied to gain a complete understanding of solid-adsorbate interactions in heterogenous systems. This limitation is partly a consequence of the complexity of surfaces in terms of both structural geometries and electronic properties. Nevertheless, some early studies have shown that FMO theory can be used to explore solid-adsorbate systems. ${ }^{5-14}$ For instance, Hoffmann et al. ${ }^{15-18}$ employed concepts of FMO theory and orbital interactions to gain information about surface-adsorbate interactions on transition metal surfaces. In very early studies, Varma and Wilson ${ }^{19}$ found that the chemisorption energy of simple molecule over metal surfaces can be described by a $d$ band model, which contains the essential parameters of transition metals such as the mean d-electron energy, the d-band width, and the number of $d$ electrons. The hybridization of adatom orbitals with the surface metal orbitals can significantly alter the local density of states of the metal atoms, and correspondingly the primary binding interaction is determined by the average position of the $d$ band with respect to the adatom orbital energy and the over width of $d$ band. More recently, Hammer and Nørskov²0 
extended and popularized the "d-band center theory", which is a widely used and versatile indicator (descriptor) of a variety of catalytic reactions occurring on transition metal (alloy) surfaces. ${ }^{21,22}$ In the dband center theory model, the higher the d-states are in energy relative to the Fermi level, the emptier the antibonding states and the stronger the adsorption energy of an adsorbed species on a transition metal (alloy) surface. Despite its extensive use in assessing reactions on transition metal surfaces, the applicability of such analysis based on the FMO theory to treating processes occurring on transition metal oxide surfaces, ${ }^{23-31}$ which have substantially different structures and properties, ${ }^{27,32-34}$ remains challenging.

In a recent investigation, we explored adsorption of various small molecules on the anatase (101) and rutile (110) surfaces of $\mathrm{TiO}_{2}$ by employing density functional theory (DFT) calculations. ${ }^{35}$ Machinelearning $(\mathrm{ML})$ based statistical methods were also used to identify descriptors (indicators) that govern the adsorption processes. The results of this effort showed that, although complicated at first sight, these types of molecular adsorption systems can be effectively treated using FMO theory. Specifically, we found that linear relationships exist between $\mathrm{HOMO}$ energies of adsorbates and their adsorption energies $\left(E_{\text {ads }}\right)$. The results of this analysis indicated that interactions between conduction bands (CBs) on surfaces and HOMOs of molecules likely play an important role in governing surface adsorption.

While serving as an important step in exploring the use of FMO theory to understanding/predicting adsorption on oxide surfaces, our previous study was performed using only a limited number of metal oxides. To obtain greater insight into the use of FMO theory in treating solid-adsorbate interactions, we designed and conducted the systematic investigation described below to explore a greater number of metal oxide surfaces. In this effort, DFT calculations and FMO analyses were performed to understand interactions occurring between a number of simple molecules (adsorbates) and surfaces of group 13 metal oxides, including $\mathrm{Al}_{2} \mathrm{O}_{3}, \mathrm{Ga}_{2} \mathrm{O}_{3}$, and $\mathrm{In}_{2} \mathrm{O}_{3}$. The group 13 metal oxides were chosen because they serve as excellent catalysts and catalyst supports for a variety of valuable chemical transformation. ${ }^{36-43}$ The results of this effort show that $E_{\text {ads }}$ for these catalysts correlate linearly with HOMO energies of the small molecule adsorbates and that they do not correlate strongly with adsorbate LUMO energies. The 
results of ML based statistical analysis demonstrate that the HOMO energies and dipole moments of adsorbates play important roles in governing molecular adsorption. In addition, surface energy is identified to be an important parameter determining the $E_{\text {ads. }}$. Because adsorption is a fundamental step in surface catalyzed reactions and it is directly related to activation of reactants, observations made in this study give important insight into understanding and predicting reactivities of the molecules on surfaces of heterogeneous catalysts.

\section{COMPUTATIONAL METHOD}

\subsection{DFT calculations}

Periodic DFT calculations were performed using the Vienna ab initio simulation package (VASP, version 5.4.4).44,45 The functional of Perdew-Burke-Ernzerhof revised for solids (PBEsol) ${ }^{46}$ was used in combination with the projector-augmented wave method. ${ }^{47} \mathrm{~A}$ kinetic energy cutoff of $400 \mathrm{eV}$ was set for the plane-wave basis sets. Gaussian smearing with a width of $0.2 \mathrm{eV}$ was applied for the occupation of the electronic levels. The Brillouin zone was sampled with Monkhorst-Pack grids of $4 \times 4 \times 1$. Van der Waals interactions were described by using the dispersion-corrected DFT-D3 (BJ). ${ }^{48}$ The convergence of force on each atom was set to $0.03 \mathrm{eV} \AA^{-1}$.

\subsection{Surface models}

The surfaces of group 13 metal oxides including $\beta-\mathrm{Ga}_{2} \mathrm{O}_{3}(100), \beta-\mathrm{Ga}_{2} \mathrm{O}_{3}(001), \theta-\mathrm{Al}_{2} \mathrm{O}_{3}(100)$, $\theta$ $\mathrm{Al}_{2} \mathrm{O}_{3}(001), \theta-\mathrm{Al}_{2} \mathrm{O}_{3}(010), \ln _{2} \mathrm{O}_{3}(110)$, and $\ln _{2} \mathrm{O}_{3}(111)$ were simulated by using supercell slab models (Figure 1). The slab models contain four to six sublayers composed of $\mathrm{M}_{2} \mathrm{O}_{3}$ unit $(\mathrm{M}=\mathrm{Ga}, \mathrm{Al}$, or In). The repeated slabs along the surface normal direction are separated by a minimum vacuum region thickness of $15 \AA$ (see Figure S1 for the side views of the slab models). The top layers with adsorbates were allowed to relax during geometry optimizations, while the bottom layers were fixed at their original bulk positions. Detailed information about the compositions of the slab models and the treatment of atomic constraints during geometry optimizations are provided in Table S1. The adsorption energy ( $\left.E_{\text {ads }}\right)$ of an adsorbate on metal oxide surface is defined as 
$E_{\mathrm{ads}}=E_{\mathrm{A} / \mathrm{S}}-E_{\mathrm{A}}-E_{\mathrm{S}}$

where $E_{\mathrm{A} / \mathrm{S}}, E_{\mathrm{A}}$, and $E_{\mathrm{S}}$ are the electronic energies of adsorption complex, free-state adsorbate, and bare metal oxide surface, respectively. The VASP calculated electronic energies of $E_{\mathrm{A} / \mathrm{S}}, E_{\mathrm{A}}$, and $E_{\mathrm{S}}$ using PBEsol functional as well as the corresponding $E_{\text {ads }}$ are provided in Tables S2 and S3. In the discussion of the $E_{\text {ads }}$ results, we referred to the values without zero-point energy (ZPE) corrections. The evaluation of ZPE contributions over $\beta-\mathrm{Ga}_{2} \mathrm{O}_{3}(100)$ surface shows the values of ZPE corrections to the $E_{\text {ads }}$ are normally less than $0.1 \mathrm{eV}$ (Table S4 and Figure S2), and the ZPE corrected and uncorrected $E_{\text {ads }}$ give a strong linear correlation $\left(R^{2}=1.00\right)$, which suggests that the exploration for the parameters correlating with $E_{\text {ads }}$ will be insignificantly affected by neglecting ZPE corrections. 

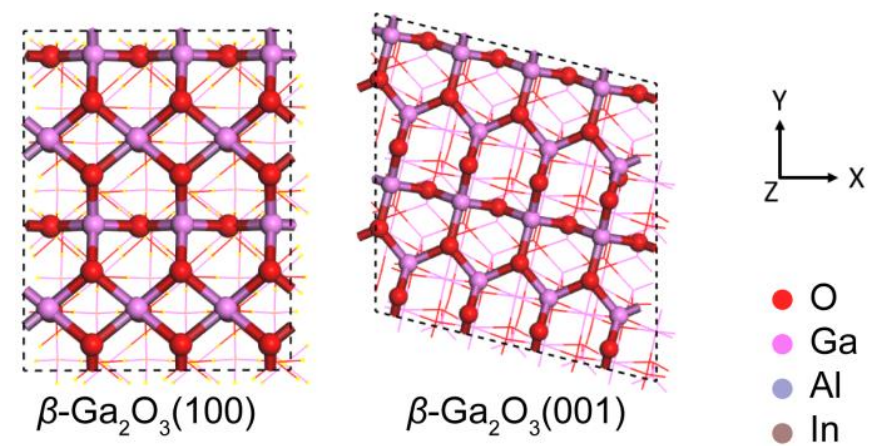

- 0

- Ga

- $\mathrm{Al}$

- In

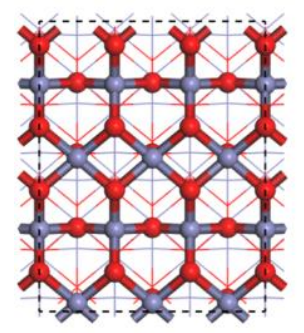

$\theta-\mathrm{Al}_{2} \mathrm{O}_{3}(100)$
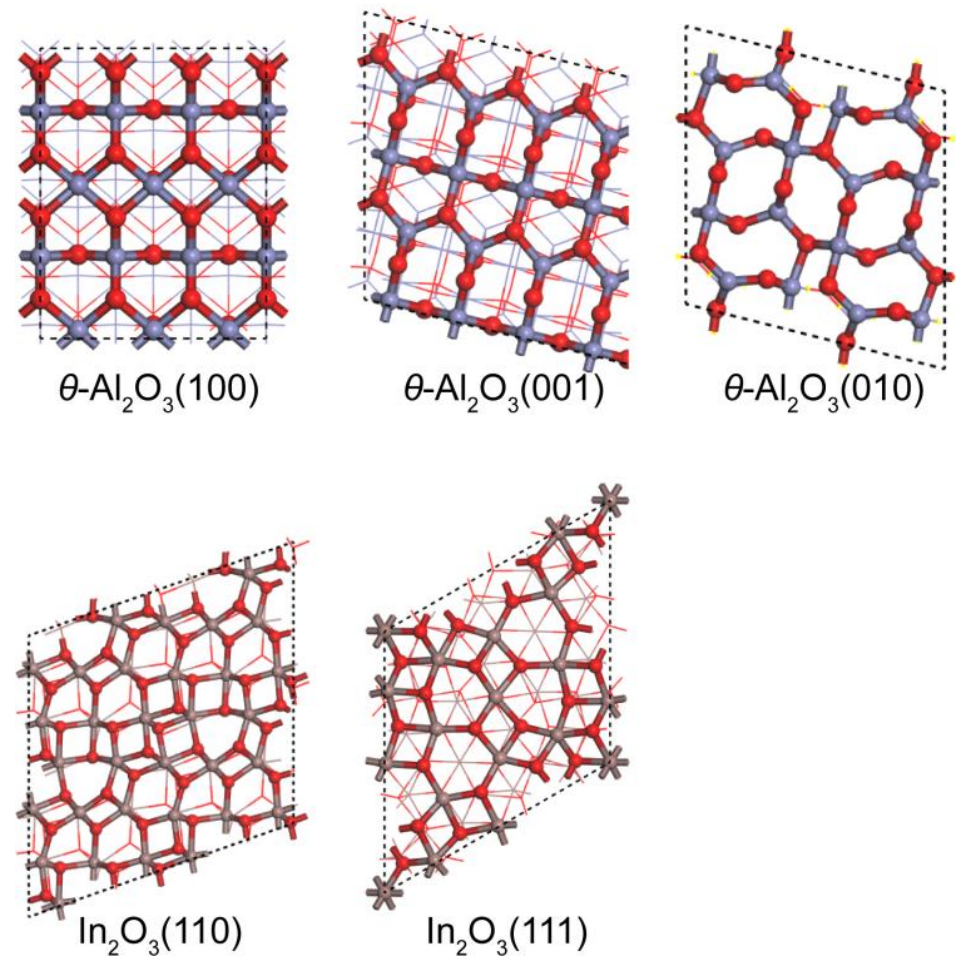

Figure 1. Slab models used for DFT calculations of group 13 metal oxide surfaces (top views). The slab models contain four to six $\mathrm{M}_{2} \mathrm{O}_{3}$ unit ( $\mathrm{M}=\mathrm{Ga}$, $\mathrm{Al}$, or In element) sublayers, and the repeated slabs along the surface normal z-direction are separated by a minimum vacuum thickness of $15 \AA$. 
2.3 Frontier orbitals of small molecule adsorbates

A variety of small molecules were utilized to investigate adsorbate-surface interaction. The list includes $\mathrm{CO}, \mathrm{CO}_{2}, \mathrm{~N}_{2}, \mathrm{NH}_{3}, \mathrm{H}_{2} \mathrm{O}$, acetonitrile, acetone, acetamide, acetic acid, alkanes, alkenes, aromatic compounds, alcohols, and amines. To obtain meaningful orbital energies of isolated molecules from periodic DFT calculations, it is essential to choose appropriate reference states. In a previous study, ${ }^{35}$ we compared HOMOs and LUMOs of various small molecules generated by using three different computational methods including (1) nonperiodic DFT computations of isolated molecules to produce benchmark data, (2) periodic DFT computations of isolated molecules contained within a large cubic unit cell where the FMOs are calculated at the vacuum level, and (3) periodic DFT calculations using a slab model of metal oxide surface. In the latter case, an anatase $\mathrm{TiO}_{2}(101)$ surface was employed and one atom of the target molecule was fixed at least $5 \AA$ from surface during geometry optimization and the FMOs were calculated from the valence band maximum (VBM) of the $\mathrm{TiO}_{2}$ surface. The nonperiodic calculations were performed at PBE/6-311G(d,p) level of theory using the Gaussian 09 program, ${ }^{49}$ while the periodic calculations were carried out using PBEsol functional combined with the projectoraugmented wave method as implemented in VASP program. ${ }^{44,45}$ The detailed calculation procedures and the computed values of HOMO and LUMO levels by three different methods were provided in ref. 35. The results of the earlier effort showed that the HOMO (or LUMO) energies of isolated molecules obtained using these three approaches possess strong linear correlations. Thus, it is feasible to use HOMOs and LUMOs arising from periodic DFT computations with an energy reference to vacuum or the VBM level of metal oxide surfaces for investigating molecular adsorption.

In the current study, the correlation of $E_{\text {ads }}$ with FMO levels were analyzed using the $\mathrm{HOMO}$ and LUMO energies (Table S3) taken from ref. 35. These values were obtained from periodic DFT calculations using PBEsol functional with projector-augmented wave potentials, in which the target molecule was placed in a large empty unit cell. To check the reliability of DFT-calculated HOMO energies, we further compared these values with the experimentally measured ionization energy (IP) of gas-phase 
molecules (Table S5). The plot of the negative of the IP (-IP) against HOMO energies calculated with PBEsol functional (Figure S3) shows a satisfactory correction $\left(R^{2}=0.88\right)$, which suggests it is feasible to use the DFT-calculated HOMO energies for exploring the correlation relationship with $E_{\text {ads. }}$ As the accessible experimental data of electron affinity (EA) are very limited, we did not provide the comparison of DFT-calculated LUMO energies with EAs. A plot of HOMO versus LUMO energies calculated by PBEsol functional (Figure S4) shows that no significant correlation exists between these two energy parameters for each small molecule, suggesting that HOMO and LUMO energies are independent properties that can be used as separate molecular descriptors for latter ML based statistical analysis.

\subsection{Machine learning (ML)}

A widely used package, scikit-learn (http://scikit-learn.org, version 0.22.1.post1), was employed for ML implementations. ${ }^{50}$ Extra trees regression (ETR), a tree ensemble method (see below for the detailed information), was used to predict $E_{\text {ads }}$ and to identify the relative importance of the variables for the prediction. ${ }^{35,51,52}$ The hyperparameter used is $n \_$estimators $=200$. Default values of scikit-learn were used for other hyperparameters. Monte Carlo cross validation with 100-times of random leave-20\%-out trials was used for evaluating the prediction performance. The root mean square error (RMSE) and the coefficient of determination $\left(\mathrm{R}^{2}\right)$ between the predicted and true values (ground truth) are calculated for each trial, and averaged to obtain the mean RMSE and $\mathrm{R}^{2}$ values. Dragon program (Kode srl, Dragon (Software for Molecular Descriptor Calculation), version 7.0.6. https://chm.kode-solutions.net, 2016.) was used to provide descriptors of molecules adsorbed. Surface energies were calculated by using the DFT method $^{53}$ and applied as descriptors.

Tree ensemble methods make prediction by combining multiple regression trees into an ensemble. A single regression tree represents a simple piece-wise constant function, and an ensemble of multiples regression trees improves the flexibility of models though it is still a piece-wise constant function. These approaches are widely known ${ }^{54,55}$ to be applicable to treatment of general high-dimensional data. In addition, compared to other methods such as the kernel methods, tree ensemble methods are less 
dependent on hyperparameter settings and, therefore, they can be utilized reliably by even non ML experts. Furthermore, tree ensemble methods also provide information about the degree to which each descriptor contributes to the prediction in the form of an importance score. ${ }^{54}$ Linear methods, such as ordinary linear regression by least squares (OLR) and least absolute shrinkage and selection operator (LASSO) regressions, give similar coefficient of descriptor outputs, but they require the assumption that a linear relationship between the target and descriptors. The importance scores of tree ensemble methods are nonlinear alternatives to the coefficients on each descriptor arising from the linear methods for evaluation of the contributions of individual descriptors to the prediction target.

\section{RESULTS AND DISCUSSION}

\subsection{Molecular adsorption on $\beta-\mathrm{Ga}_{2} \mathrm{O}_{3}(100)$}

Because it has served as the focus of earlier studies, ${ }^{56-59}$ the $\beta-\mathrm{Ga}_{2} \mathrm{O}_{3}(100)$ surface was utilized as a representative example in our initial discussions of adsorption of small molecules on metal oxides. Several possible adsorption sites were considered when selecting the most stable adsorption configuration that should be used to determine the $E_{\text {ads }}$ of an adsorbate. The respective optimized structures for small molecules adsorbed to the $\beta-\mathrm{Ga}_{2} \mathrm{O}_{3}(100)$ surface and calculated $E_{\text {ads }}$ are given in Figure S5 and Table S3. To search for the existence of correlations between $E_{\text {ads }}$ and FMO energies of the adsorbates, plots of $E_{\text {ads }}$ versus $\mathrm{HOMO}$ energies were constructed (Figure 2a). Indeed, inspection of these plots suggests that a linear correlation with a $R^{2}$ value of 0.66 does exist between these two parameters. In contrast, when computed at the present GGA/PBEsol level of theory, the $E_{\text {ads }}$ values do not display a linear correlation with LUMO energies of the adsorbates $\left(R^{2}=0.00\right)$ (Figure $\left.2 c\right)$. These

findings are in accord with a simple consideration of Lewis acid-base theory, ${ }^{23,60,61}$ which suggests that small molecules serve as Lewis bases acting as electron pair-donors, and thus it is expected the frontier orbital occupied by elections (i.e., HOMO) rather than LUMO plays a main role in the adsorbate-surface interaction. 

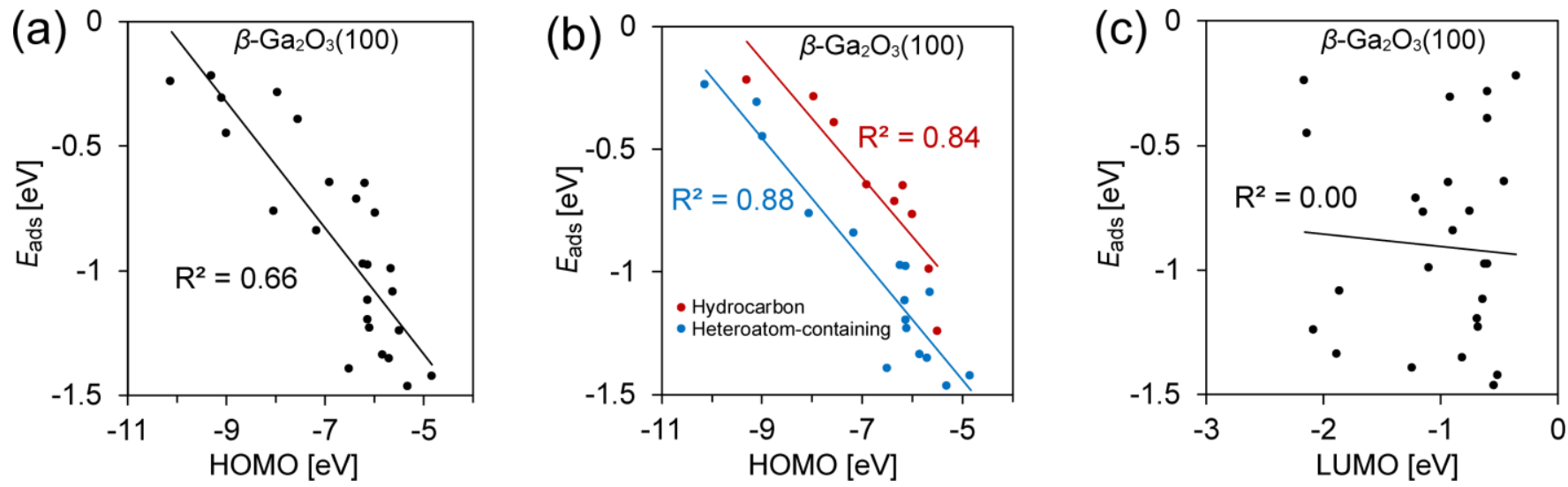

Figure 2. Correlation of $E_{\text {ads }}$ with $\mathrm{HOMO}$ energies of all small molecules (a) and of hydrocarbons and heteroatom-containing small molecules (b), and LUMO energies of all small molecules (c) adsorbed on $\beta-\mathrm{Ga}_{2} \mathrm{O}_{3}(100)$ surface. All the considered adsorbates and the calculated values of $E_{\text {ads }}$ and $\mathrm{HOMO} / \mathrm{LUMO}$ energies are provided in Table S3.

Moreover, separate plots of $E_{\text {ads }}$ versus HOMO energies for two groups of small molecules classified as hydrocarbons and heteroatom-containing compounds (Figure 2b) have higher linear correlations $\left(R^{2}=0.84\right.$ for hydrocarbons and $R^{2}=0.88$ for heteroatom-containing compounds) than the one in which all molecules are included in one group. This is likely a consequence of differences in the nature of orbital interactions associated with adsorption where heteroatom-containing compounds normally utilize lone electron pair containing orbitals to form chemical bonds with surface Lewis acid sites on metals, while hydrocarbon adsorption is strongly influenced by van der Waals (vdW) interactions. ${ }^{35,62}$ A plot of $E_{\text {ads }}$ versus $\mathrm{HOMO}$ energies for nine selected hydrocarbons (Figure 3 ) shows that vdW interactions, which increase as the molecular size of the hydrocarbon increases, is a large contributor to $E_{\text {ads. }}$ Because vdW interactions are not directly related to FMO interactions, we speculate that the observed linear correlation seen in the $E_{\text {ads }}$ versus $\mathrm{HOMO}$ energy plot for hydrocarbons is a coincidental effect of molecular size on HOMO energy trends. In any event, the FMO data should still be useful in establishing preliminarily trends to estimate the $E_{\text {ads }}$ of small molecules and to perform ML-based statistical analysis. 


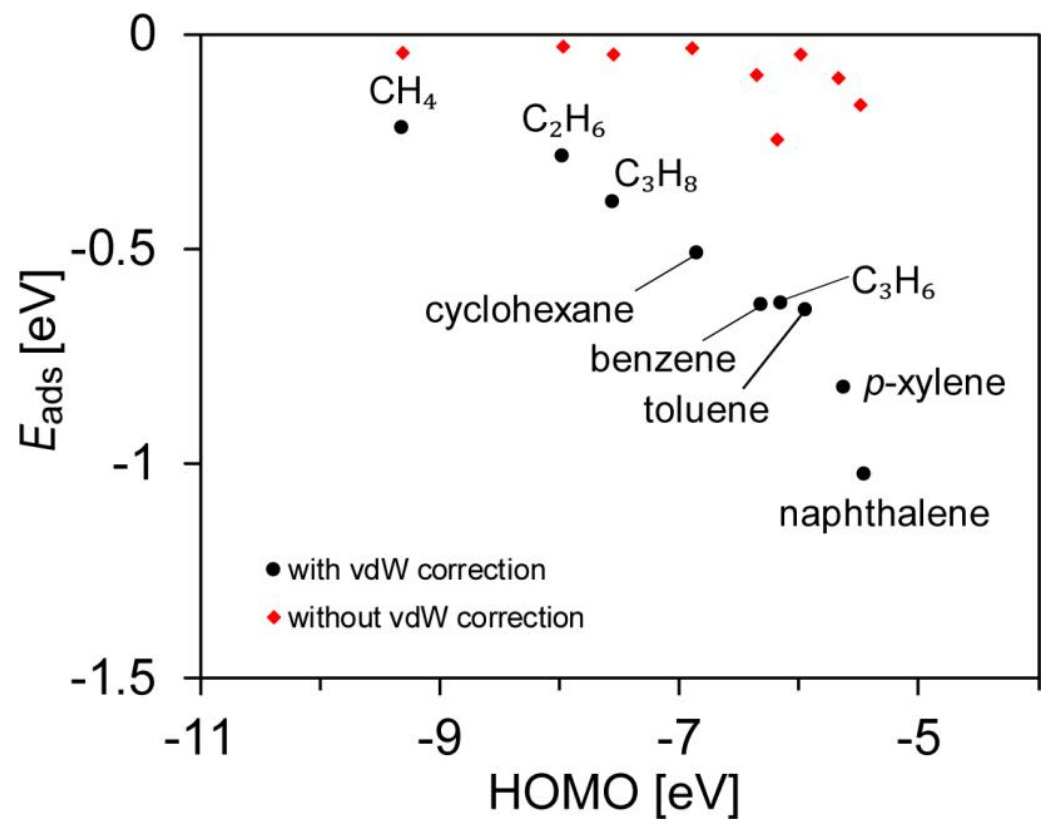

Figure 3. HOMO energies vs $E_{\text {ads }}$ for selected hydrocarbons showing the effect of van der Waals/dispersion interactions on adsorption to $\beta-\mathrm{Ga}_{2} \mathrm{O}_{3}(100)$. The values of vdW correlated and uncorrected $E_{\text {ads }}$ were computed with and without vdW-energy described by Grimme's D3 correction method. 48

To evaluate the influence of DFT functionals on the computed $E_{\text {ads }}$, we further employed PBE ${ }^{63}$ and revPBE ${ }^{64}$ functionals as implemented in VASP program. ${ }^{44,45}$ The obtained values of $E_{\text {ads }}$ were provided in Table S6. The comparison with those as determined by PBEsol indicates that the values of $E_{\text {ads }}$ are strongly affected by DFT functionals. However, as shown in Figure S6, the PBEsol calculated $E_{\text {ads }}$ show a strong correlation with those computed by either PBE $\left(R^{2}=1\right)$ or revPBE $\left(R^{2}=0.94\right)$. This is possibly due to the error calculations in different DFT methods. As the computed $E_{\text {ads }}$ using different functionals are strongly correlated, we assume the choice of different functionals will not affect our conclusion on the linear correlation between $E_{\text {ads }}$ and $\mathrm{HOMO}$ energies.

\subsection{Molecular adsorption on other group 13 metal oxide surfaces}


Figure 4 shows plots of HOMO and LUMO energies of small molecules vs. $E_{\text {ads }}$ values for adsorption to surfaces of other group 13 metal oxides, including $\beta-\mathrm{Ga}_{2} \mathrm{O}_{3}(001), \quad \theta-\mathrm{Al}_{2} \mathrm{O}_{3}(100), \quad \theta-\mathrm{Al}_{2} \mathrm{O}_{3}(001), \quad \theta-$ $\mathrm{Al}_{2} \mathrm{O}_{3}(010), \mathrm{In}_{2} \mathrm{O}_{3}(110)$, and $\ln _{2} \mathrm{O}_{3}(111)$. These plots show correlations that are similar to those seen for adsorption on $\beta-\mathrm{Ga}_{2} \mathrm{O}_{3}(001)$. Specifically, $E_{\text {ads }}$ values linearly correlate with $\mathrm{HOMO}$ energies of the adsorbates and they do not display linear correlation with LUMO energies when computed at the present GGA/PBEsol level of theory. Also, better correlations are obtained by separately treating data for hydrocarbons and heteroatom-containing compounds. Inspection of slope, intercept and $\mathrm{R}^{2}$ data obtained by linear regression analysis of the $E_{\text {ads }}$ vs HOMO energy plots (Table S7) suggests that the effect of HOMO energies on adsorption depend on the nature of the metal oxide and they vary significantly when the crystallographic planes are changed.

For the molecular adsorption over isostructural surfaces, i.e., $\beta-\mathrm{Ga}_{2} \mathrm{O}_{3}(100)$ vs. $\theta-\mathrm{Al}_{2} \mathrm{O}_{3}(100)$ or $\beta$ $\mathrm{Ga}_{2} \mathrm{O}_{3}(001)$ vs. $\theta-\mathrm{Al}_{2} \mathrm{O}_{3}(001)$, the variation from $\mathrm{Ga}$ to $\mathrm{Al}$ does not afford a consistent trend in the adsorption strength for the investigated molecules (Table S3). The $E_{\text {ads }}$ differences of these adsorbates are generally less than $0.1 \mathrm{eV}$, which suggests the change of metal element does not afford a significantly large variation in the $E_{\text {ads }}$ with the scope of isostructural oxide surfaces considered in this study. 
(a)

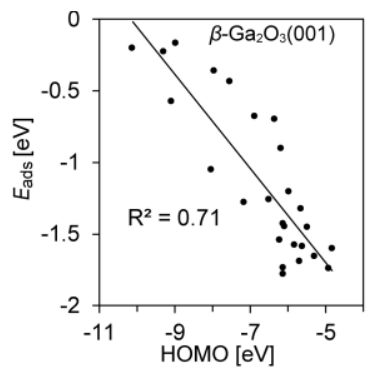

(b)

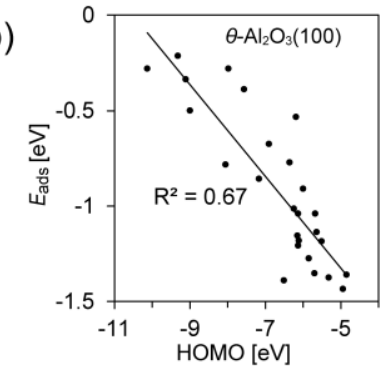

(c)

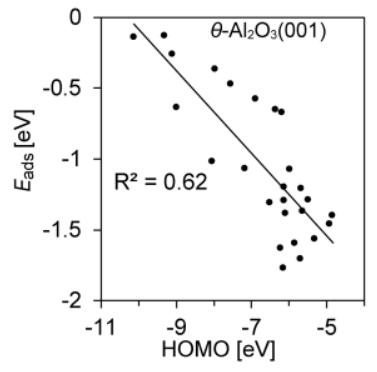

(d)

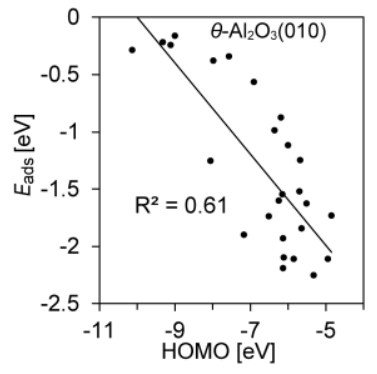

(e)

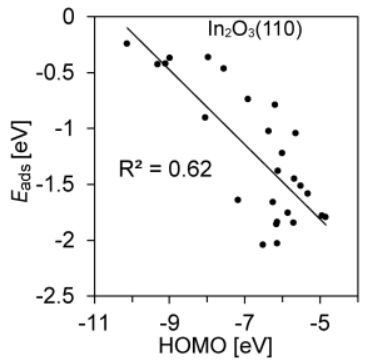

(f)

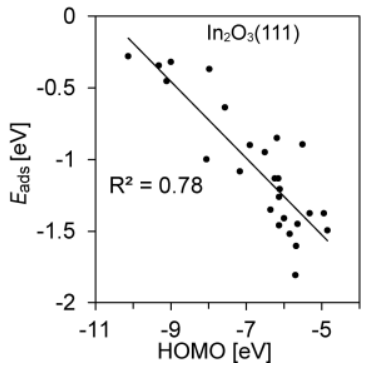

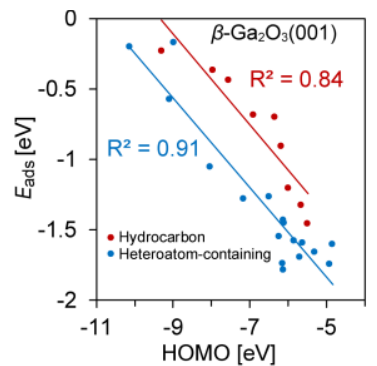
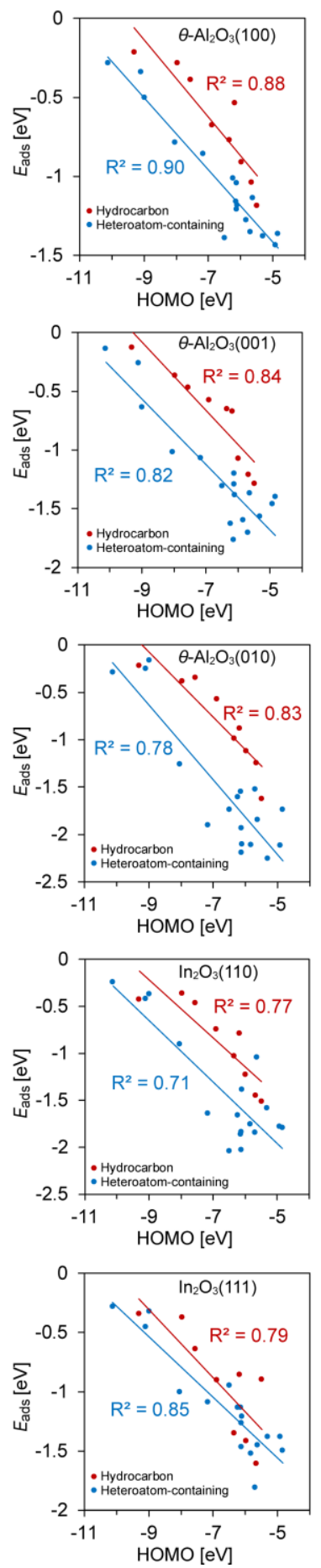
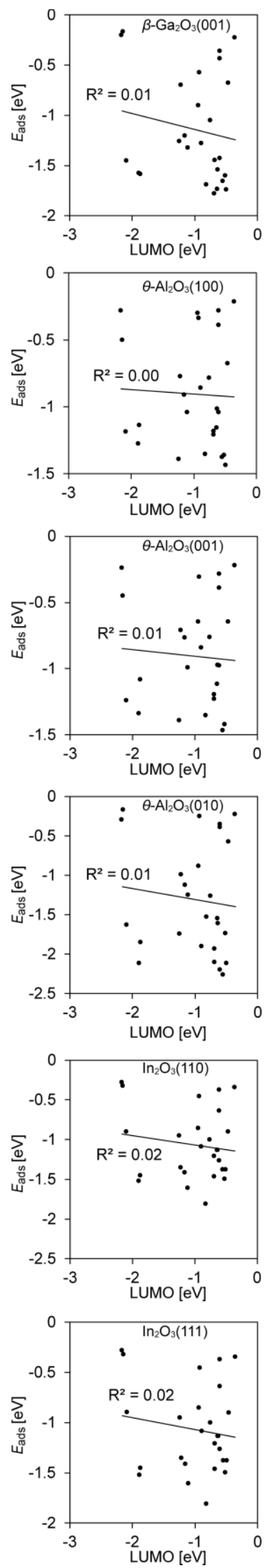
Figure 4. Correlation of $E_{\text {ads }}$ with HOMO and LUMO energies of small molecules adsorbed on (a) $\beta$ $\mathrm{Ga}_{2} \mathrm{O}_{3}(001)$, (b) $\theta-\mathrm{Al}_{2} \mathrm{O}_{3}(100)$, (c) $\theta-\mathrm{Al}_{2} \mathrm{O}_{3}(001)$, (d) $\theta-\mathrm{Al}_{2} \mathrm{O}_{3}(010)$, (e) $\ln _{2} \mathrm{O}_{3}(110)$, and (f) $\ln _{2} \mathrm{O}_{3}(111)$. All the considered adsorbates and the calculated values of $E_{\text {ads }}$ and $\mathrm{HOMO} / \mathrm{LUMO}$ energies are provided in Table S3.

Surface energy is considered to be a factor that governs $E_{\text {ads }}$ values for adsorption of molecules on metal oxide surfaces. ${ }^{35}$ Adsorption of a single molecule on different surfaces should afford $E_{\text {ads }}$ values that serve as parameters that can be used in statistical analysis of the surface energies and related adsorption behaviors of metal oxide surfaces. The usage of surface energy to describe the adsorption activity may be related to its ability to reflect the degree of coordinative unsaturation of the surface atoms. ${ }^{65}$ In the next phase of the current effort, we explored this issue using acetonitrile as a test molecule to interrogate the properties of group 13 metal oxide surfaces. A major factor governing bonding of acetonitrile to metal surfaces is interaction of the terminal cyano nitrogen with Lewis acid metal sites. The respective $E_{\text {ads }}$ values of $-0.74,-1.05,-0.76,-1.02,-1.24,-0.90,-1.00 \mathrm{eV}$ for adsorption to $\beta$ $\mathrm{Ga}_{2} \mathrm{O}_{3}(100), \beta-\mathrm{Ga}_{2} \mathrm{O}_{3}(001), \theta-\mathrm{Al}_{2} \mathrm{O}_{3}(100), \theta-\mathrm{Al}_{2} \mathrm{O}_{3}(001), \theta-\mathrm{Al}_{2} \mathrm{O}_{3}(010), \ln _{2} \mathrm{O}_{3}(110)$, and $\ln _{2} \mathrm{O}_{3}(111)$ were employed to construct the $E_{\text {ads }}$ vs surface energy plot displayed in Figure 5, which shows that a strong linear correlation $\left(R^{2}=0.88\right)$ exists between these properties. Accordingly, as the surface energy of the metal oxide increases the adsorption of acetonitrile becomes stronger. We further analyzed the effect of surface energy on the slopes of linear regression equations for different metal oxide surfaces (Figure S7), which indicates that a higher surface energy affords a more negative value of slope for the metal oxide surfaces with same elements. This may be because a higher surface energy provides a stronger molecular attraction, which can stabilize the adsorption system to a larger extent, i.e., a more negative slope. 


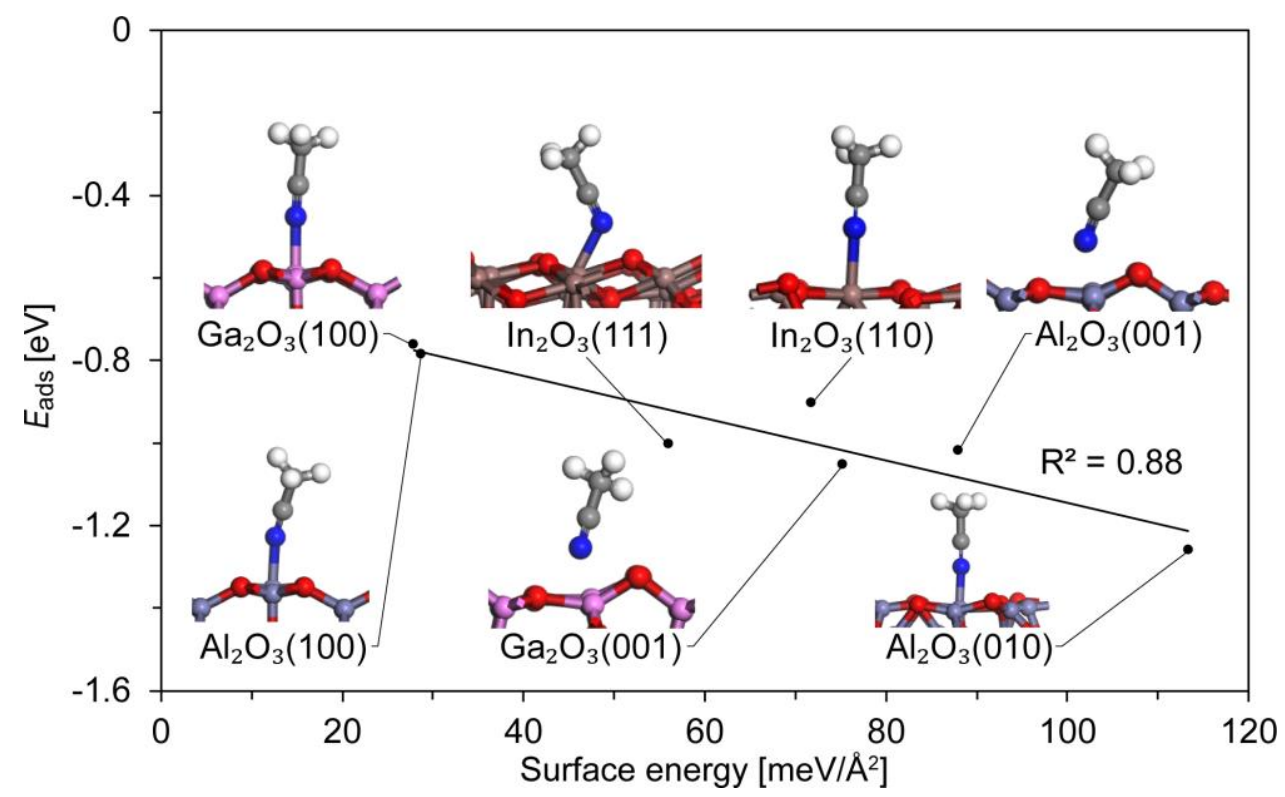

Figure 5. Plot of $E_{\text {ads }}$ values for adsorption of acetonitrile vs metal oxide surface energies. The surface energy is defined as $\left(E_{\text {slab }}-E_{\text {bulk }}\right) / 2 \mathrm{~A}$, where $E_{\text {slab }}$ and $E_{\text {bulk }}$ are the electronic energy of the slab and the bulk unit cell, and $A$ is the surface area of the slab (Table S8).

\subsection{Interaction of small molecule FMOs with metal oxide surfaces}

Overlap of atomic orbitals in semiconducting/insulating oxides results in the formation of quantized energy states, which are separated by a band gap into lower energy electron occupied valence bands (VBs) and higher energy empty CBs. In molecular adsorption on such solid surfaces, stabilization occurs as a consequence of interactions of FMOs of adsorbates with metal oxide VBs and CBs. As an approximation, main contributions to stabilization arise from HOMO-CB or LUMO-VB interactions, ${ }^{66}$ in a manner that depends on the intrinsic properties of the adsorbates and surfaces. HOMO-CB interactions are associated with electron transfer from HOMOs of adsorbates to empty surface orbitals, while LUMOVB interactions are a reverse process in which electrons are transferred from surfaces to adsorbate LUMOs. According to FMO theory, the energy difference between two interacting orbitals strongly affects the strength of the orbital interaction, and a smaller difference results in a stronger interaction in the case of a filled orbital interacting with an unfilled orbital. 
For discussion purposes, we have approximated the energies of $C B$ and VB in the $\beta-\mathrm{Ga}_{2} \mathrm{O}_{3}(100)$ surface model using energies of the CBM and the VBM as being $+1.80 \mathrm{eV}$ and $-0.46 \mathrm{eV}$, respectively. Figure 6 shows the density of states (DOS) for the $\beta-\mathrm{Ga}_{2} \mathrm{O}_{3}(100)$ surface model and the frontier orbital energies of selected small molecule adsorbates. In general, the differences in energies between the HOMOs of small molecules and the CBM of $\beta-\mathrm{Ga}_{2} \mathrm{O}_{3}(100)$ are smaller than those between LUMOs and the CBM. This trend is particularly noticeable for molecules that strongly adsorb to the surface $\beta$ $\mathrm{Ga}_{2} \mathrm{O}_{3}(100)$. For example, in the case of amines like methyl-, dimethyl- and trimethylamine ( $E_{\mathrm{ads}}=-1.44$, -1.46 , and $-1.34 \mathrm{eV}$, respectively), the respective HOMO-CBM (LUMO-VBM) energy differences are 0.48 (7.36), -0.10 (7.42), and -0.01 (7.40) eV. Thus, HOMO-CB instead of LUMO-VB orbital interactions play a primary role in governing the strengths of solid-adsorbate interactions. The insight gained from FMO reasoning is consistent with conclusions based on Lewis acid-base theory (see above). In the adsorption systems probed in this effort, the metal oxide surface provides Lewis acid/electron pair acceptor sites, while the adsorbates serve as Lewis bases/electron donors. Therefore, it is expected that HOMO energies of adsorbates should be well correlated with adsorption energies. DOS analysis also indicates that orbital energies of adsorbed molecules are shifted with respect to those of isolated adsorbates (Figure S8), This phenomenon along with inspection of visualized electron density isosurfaces serves as evidence for interactions that occur between adsorbate and solid surface orbitals (Figure S8). 


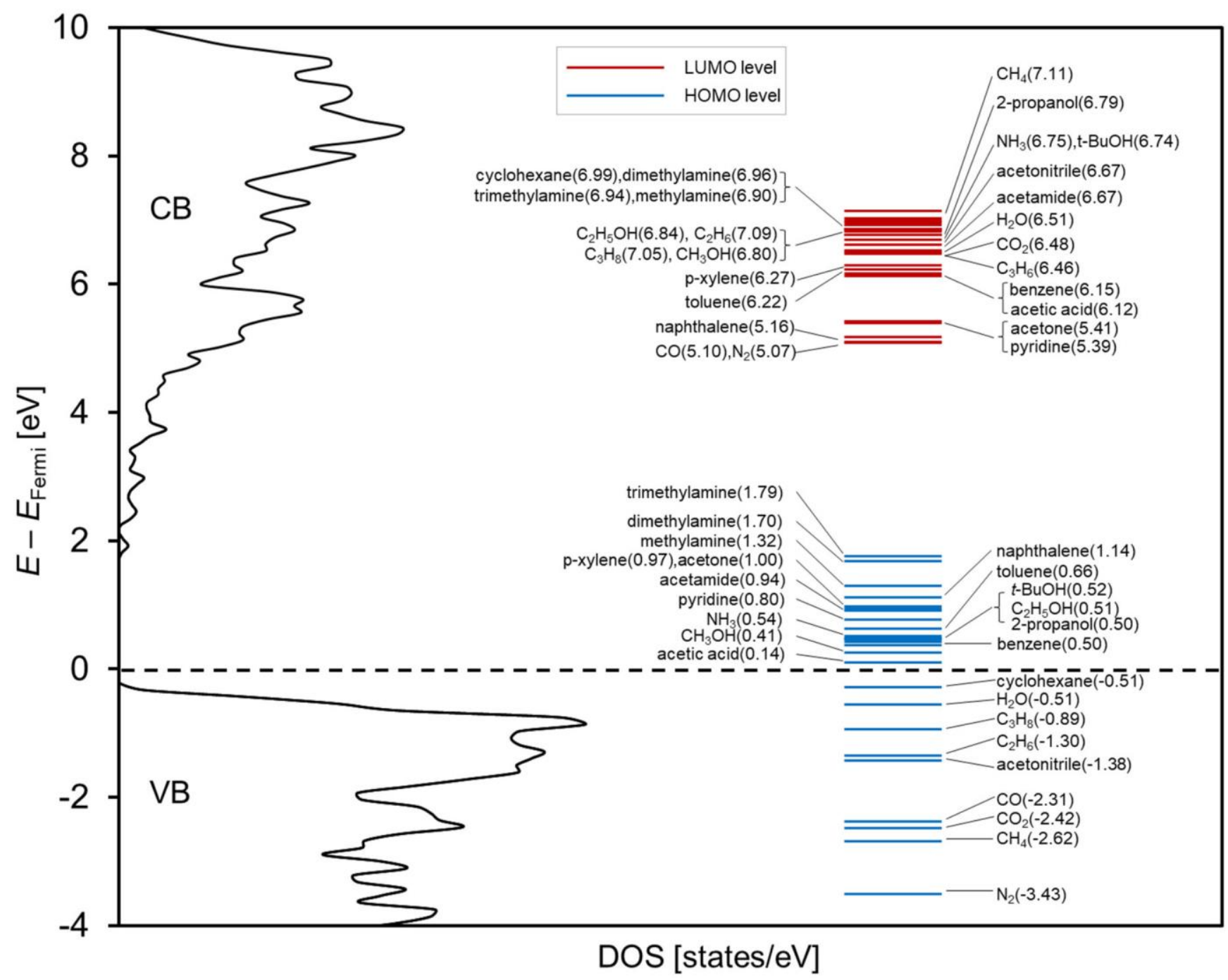

Figure 6. Density of states (DOS) for the $\beta-\mathrm{Ga}_{2} \mathrm{O}_{3}(100)$ surface model, and $\mathrm{HOMO}$ and LUMO energies of small molecule adsorbates. The DOS was calculated with the Brillouin zone sampled with $6 \times 6 \times 1 \mathrm{k}$ point meshes. The HOMO and LUMO energies are given relative to the valence band maxima (VBM) of $\beta-\mathrm{Ga}_{2} \mathrm{O}_{3}(100)$. 


\subsection{ML based statistical analysis}

The $E_{\text {ads }}$ values for adsorption of small molecules on group 13 metal oxide surfaces (Table S3) were calculated at the same level of theory, which provide a clean and consistent data set for use in ML-based statistical analysis. ML methods can not only provide platforms for predictions but also they can aid in identifying input variables (descriptors) that are important for making predictions. ${ }^{35,52,67} \mathrm{~A}$ wide range of descriptors were evaluated using the Dragon program to identify parameters that are important for predicting $E_{\text {ads }}$ for small molecule adsorption to metal oxides. A total number of 3881 very simple ones to complex descriptors were employed in the statistical analysis along with HOMO, HOMO-1 and LUMO energies of isolated adsorbates and adsorbed molecules, which have been previously shown to serve as potential quantum-chemical descriptors. ${ }^{35,68,69}$ In Figure 7 is given a map correlating orbital and surface energies, molecular weights, and dipole moments. In a manner that is consistent with the DFT calculation derived correlations in Figures 2 and 4, the $E_{\text {ads }}$ parameter strongly correlates with the HOMO energies of isolated molecules. For the orbital parameters, the HOMO energies of isolated molecules show a strong correlation with HOMO energies of adsorbed molecule, and additionally have moderate correlations with the HOMO-1 energies of isolated molecules.

To reduce multicollinearity, we selected five important descriptors, including surface energy, HOMOs and LUMOs of isolated molecules, dipole moment, and molecular weight. Then a descriptor was removed when it produces a correlation coefficient $(R)$ that is larger than 0.25 or lower than -0.25 with one of the five important descriptors. This led to the identification of 106 descriptors, which were used along with the extra-trees regressor to rate the relative importance of the parameters in predicting $E_{\text {ads. }}$ The ten most important descriptors (details in Table S9) found by using this protocol are listed in the plot given in Figure 8a. This statistical analysis clearly demonstrates that HOMO energies of isolated small molecules is the most important descriptor for predicting $E_{\text {ads }}$ values and that the descriptor of surface energies of the metal oxide adsorbents also plays an important role in predicting this property. Moreover, the results of the analysis based on the ML model also suggest that dipole moment is statistically relevant for predicting $E_{\text {ads, }}$ likely because dipole based interactions between adsorbates and surfaces also 
contributes to making the adsorption process favorable. In order to visualize the marginal effect of the descriptor values on the predicted target $\left(E_{\mathrm{ads}}\right)$ response, one-way and two-way partial dependence plots (PDPs) were computed for the top three descriptors (HOMO of isolated molecules, surface energies of metal oxide surfaces, and dipole moments of isolated molecules), as shown in Figure 9. Note that only the relationship of the HOMO values with the other descriptors are shown for two-way PDPs. Obtained results show that $E_{\text {ads }}$ (negatively) increases with increase in the HOMO energy, surface energy, and dipole moment. These analyses such as feature importance analysis and PDPs would serve as a useful tool to identify and quantify important factors (descriptors) to unravel underlying chemical/physical principles.

To test the predictive capability of the ML model, the data were separated into training and test sets using a random selection process in which $20 \%$ of the data was used as the test set. The predictive performance of the ETR method, utilizing 106 descriptors and after training, is demonstrated by the highly linear correlation seen between the $E_{\text {ads }}$ and test set DFT values $\left(R^{2}=0.89\right)$ by viewing Figure $8 b$. The observed average root mean square error (RMSE) of $0.17 \mathrm{eV}$ demonstrates the high predictive capability of the ML model. The results illustrate the potential power of using ML-based statistical analysis as a simple and useful tool for exploring important parameters governing chemical process such as molecular adsorption. 


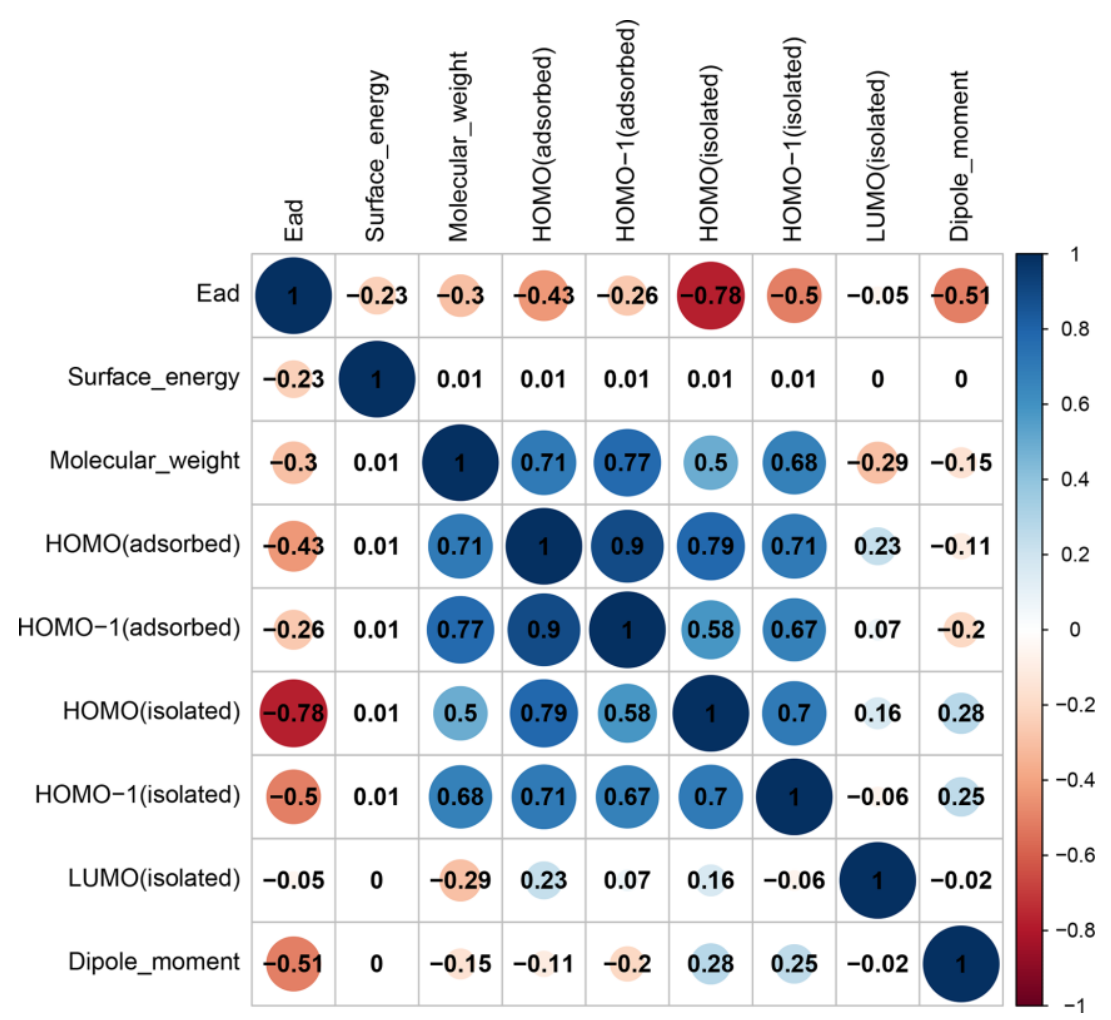

Figure 7. Map for correlation of adsorption, orbital and surface energies, molecular weights, and dipole moments. The correlation coefficients are indicated by the numbers in the circles, and a positive/negative number signifies the respective two variables change in the same/opposite direction.

(a)

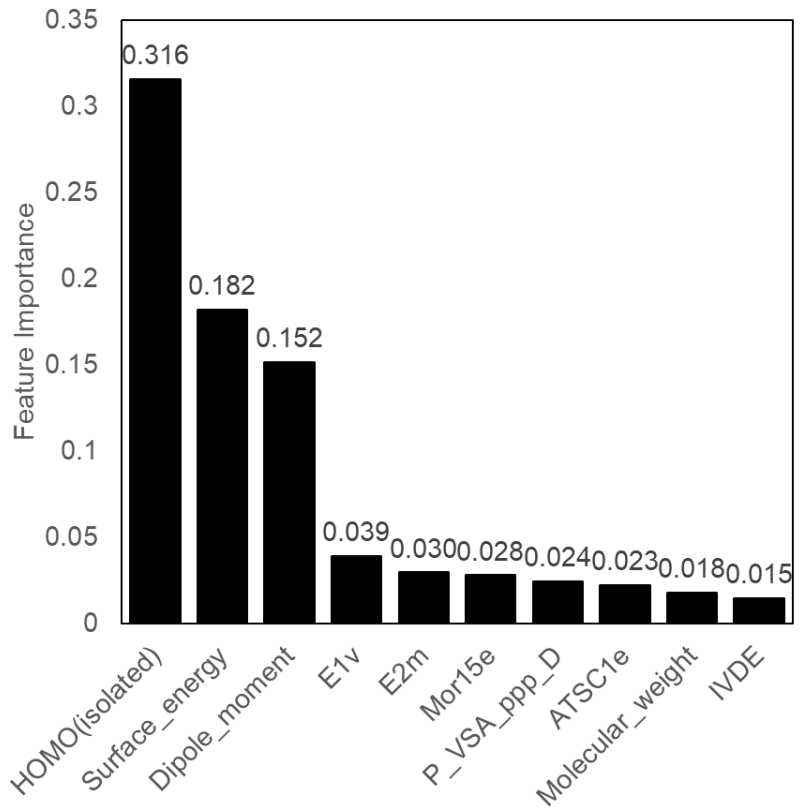

(b)

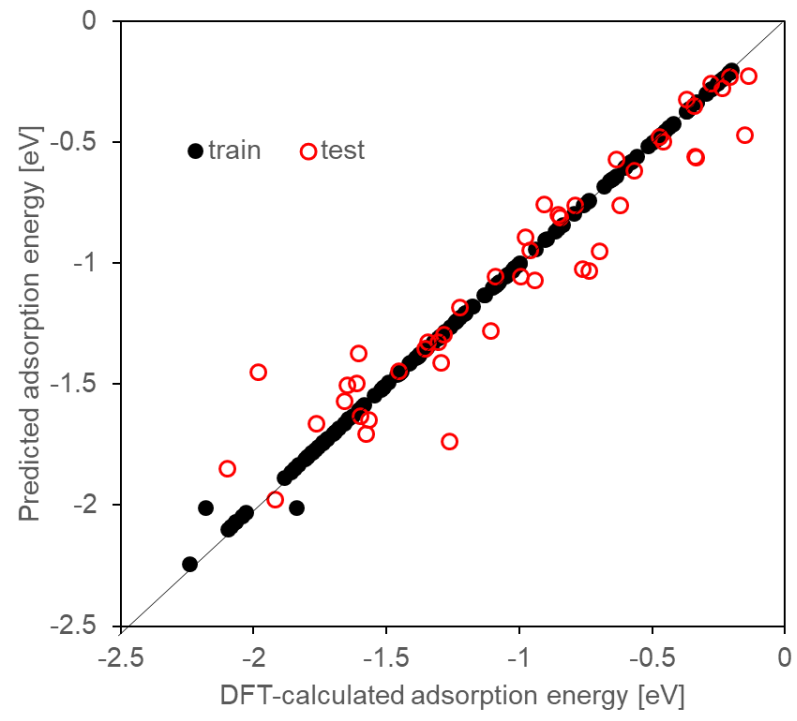


Figure 8. (a) Importance scores for high ranking descriptors obtained using extra trees regression (ETR) with 200 decision trees (Details of the highest ranked descriptors are provided in Table S9); (b) Adsorption energies of small molecules on group 13 metal oxides and values predicted using the ETR method; data in ML model were separated into training and test sets using a random selection process with $20 \%$ of the data in the test set.
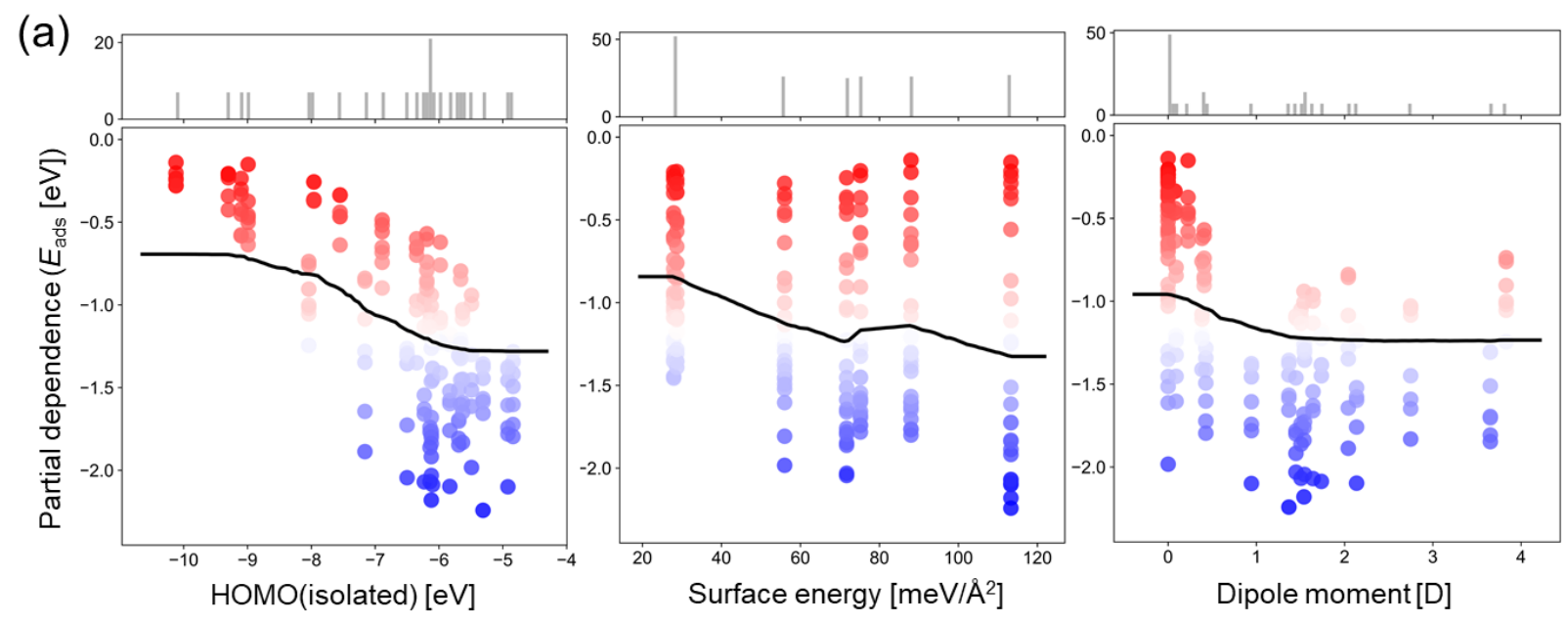

(b)
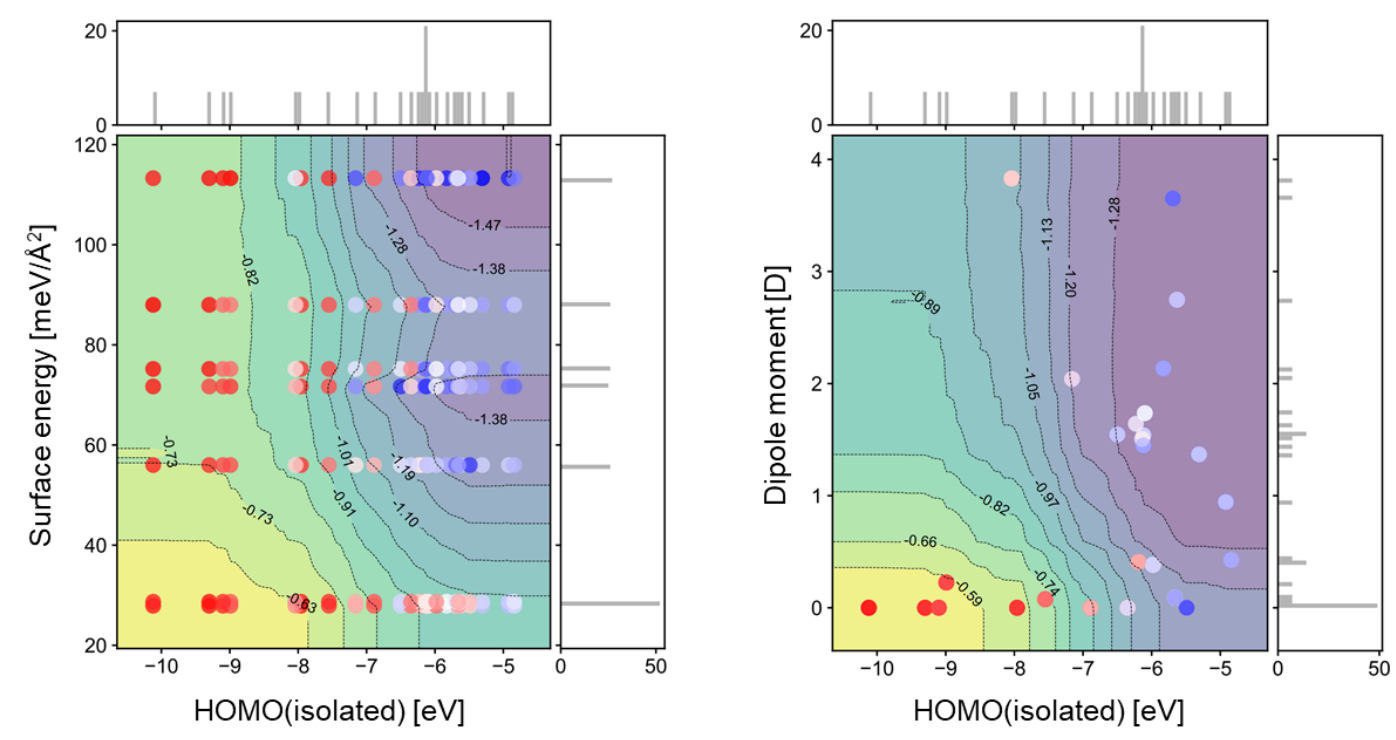

$-0.50$

$-0.75$

$-1.00$

$-1.25$

$-1.50$

$-1.75$

$-2.00$

.

Figure 9. (a) One-way (univariate) and (b) two-way (bivariate) partial dependence plots (PDPs) showing the marginal effect of the top three descriptors on $E_{\text {ads }}$ for the ETR model. The lines in (a) and contours in (b) correspond to PDPs. The histogram and scatter plots of the actual values are also shown. 


\section{CONCLUSIONS}

In the investigation described above, factors governing adsorption of common small molecules on surfaces of group 13 metal oxide surfaces, including $\beta-\mathrm{Ga}_{2} \mathrm{O}_{3}(100), \beta-\mathrm{Ga}_{2} \mathrm{O}_{3}(001), \theta-\mathrm{Al}_{2} \mathrm{O}_{3}(100), \theta-$ $\mathrm{Al}_{2} \mathrm{O}_{3}(001), \theta-\mathrm{Al}_{2} \mathrm{O}_{3}(010), \ln _{2} \mathrm{O}_{3}(110)$, and $\ln _{2} \mathrm{O}_{3}(111)$, were assessed by using DFT calculations and statistical analysis. The results show that adsorption energies $\left(E_{\mathrm{ads}}\right)$ of each metal oxide surface display good linear correlations with the HOMO energies of a variety of small molecule adsorbates while almost no correlations exist between $E_{\text {ads }}$ and the LUMO energies of the adsorbates. In addition, the results demonstrate that molecular adsorption on surfaces is mainly a consequence of interactions of HOMOs of the adsorbates with surface conduction bands of the metal oxides. Moreover, surface energies are important factors affecting small molecule adsorption on different group 13 metal oxide surfaces. Finally, the results of applying a ML approach provide additional evidence for the conclusion that adsorbate $\mathrm{HOMO}$ energies and surface energies strongly contribute to determining $E_{\mathrm{ads}}$. Additionally, they reveal that dipole moments of adsorbates also contribute to governing strengths of molecular adsorption on metal oxide surfaces. Herein we have only considered the non-dissociative molecular adsorption over the metal oxide surfaces with ideal bulk termination, and the factors dominating the adsorption process may vary for other situations such as dissociative adsorption, the presence of defects like step edges and surface oxygen vacancies, or surface reconstructions under reaction conditions. ${ }^{70}$ 


\section{AUTHOR INFORMATION}

Corresponding author

*Takashi Toyao (toyao@cat.hokudai.ac.jp)

*Ken-ichi Shimizu (kshimizu@cat.hokudai.ac.jp)

\section{ASSOCIATED CONTENT}

\section{Supporting Information}

The Supporting Information is available free of charge on the ACS Publications website at http://pubs.acs.org.

Structure models, Additional computational result, list of important descriptors for $E_{\text {ads }}$ prediction

\section{ACKNOWLEDGEMENT}

This study was financially supported by the JST-CREST project JPMJCR17J3, KAKENHI grants 17H01341, 18K14051, and 18K14057 from JSPS and by MEXT projects "Elements Strategy Initiative to Form Core Research Center" (JPMXP0112101003) and IRCCS. C.L. acknowledges support from a JSPS postdoctoral fellowship (no. P19059). A portion of the calculations was performed on supercomputers at RIIT (Kyushu Univ.) and ACCMS (Kyoto Univ.). 


\section{REFERENCES}

(1) Nørskov, J. K.; Bligaard, T.; Rossmeisl, J.; Christensen, C. H. Towards the Computational Design of Solid Catalysts. Nat. Chem. 2009, 1, 37-46. https://doi.org/10.1038/nchem.121.

(2) Schlögl, R. Heterogeneous Catalysis. Angew. Chem. Int. Ed. 2015, 54, 3465-3520. https://doi.org/10.1002/anie.201410738.

(3) Bruix, A.; Margraf, J. T.; Andersen, M.; Reuter, K. First-Principles-Based Multiscale Modelling of Heterogeneous Catalysis. Nat. Catal. 2019, 2, 659-670. https://doi.org/10.1038/s41929-019-0298-3.

(4) Fukui, K. The Role of Frontier Orbitals in Chemical Reactions (Nobel Lecture). Angew. Chem. Int. Ed. 1982, 21, 801-809. https://doi.org/10.1002/anie.198208013.

(5) Gadzuk, J. W. Surface Molecules and Chemisorption. Surf. Sci. 1974, 43, 44-60. https://doi.org/10.1016/0039-6028(74)90218-0.

(6) Wilson, A. J.; Varma, C. M. Systematics of the Binding Energy of Oxygen and Hydrogen on Transition-Metal Surfaces. II. Phys. Rev. B 1980, 22, 3805-3811. https://doi.org/10.1103/PhysRevB.22.3805.

(7) Andreoni, W.; Varma, C. M. Binding and Dissociation of CO on Transition-Metal Surfaces. Phys. Rev. B 1981, 23, 437-444. https://doi.org/10.1103/PhysRevB.23.437.

(8) Ray, N. K.; Anderson, A. B. Molecular Orbital Study of CO Chemisorption and Oxidation on a Pt(111) Surface. Surf. Sci. 1982, 112, 35-45. https://doi.org/10.1016/0039-6028(82)90185-6.

(9) Shustorovich, E.; Baetzold, R. C.; Muetterties, E. L. A Theoretical Model of Metal Surface Reactions. J. Phys. Chem. 1983, 87, 1100-1113. https://doi.org/10.1021/j100230a005.

(10) Kang, D. B.; Anderson, A. B. Theoretical Interpretation of the Cyclohexane $\rightarrow$ Benzene Reaction on the Pt(111) Surface. J. Am. Chem. Soc. 1985, 107, 7858-7861. https://doi.org/10.1021/ja00312a009.

(11) Pallassana, V.; Neurock, M. Electronic Factors Governing Ethylene Hydrogenation and Dehydrogenation Activity of Pseudomorphic PdML/Re(0001), PdML/Ru(0001), $\mathrm{Pd}(111)$, and PdML/Au(111) Surfaces. J. Catal. 2000, 191, 301-317. https://doi.org/10.1006/jcat.1999.2724.

(12) Sakong, S.; Mosch, C.; Lozano, A.; Busnengo, H. F.; Groß, A. Lowering Energy Barriers in Surface Reactions through Concerted Reaction Mechanisms. ChemPhysChem 2012, 13, 3467-3471. https://doi.org/10.1002/cphc.201200526.

(13) Huang, B.; Xiao, L.; Lu, J.; Zhuang, L. Spatially Resolved Quantification of the Surface Reactivity of Solid Catalysts. Angew. Chem. Int. Ed. 2016, 55, 6239-6243. https://doi.org/10.1002/anie.201601824.

(14) Kakekhani, A.; Roling, L. T.; Kulkarni, A.; Latimer, A. A.; Abroshan, H.; Schumann, J.; Aljama, H.; Siahrostami, S.; Ismail-Beigi, S.; Abild-Pedersen, F.; Nørskov, J. K. Nature of Lone-Pair-Surface Bonds and Their Scaling Relations. Inorg. Chem. 2018, 57, 7222-7238. https://doi.org/10.1021/acs.inorgchem.8b00902.

(15) Saillard, J. Y.; Hoffmann, R. C-H and H-H Activation in Transition Metal Complexes and on Surfaces. J. Am. Chem. Soc. 1984, 106, 2006-2026. https://doi.org/10.1021/ja00319a020.

(16) Sung, S. S.; Hoffmann, R. How Carbon Monoxide Bonds to Metal Surfaces. J. Am. Chem. Soc. 1985, 107, 
578-584. https://doi.org/10.1021/ja00289a009.

(17) Hoffmann, R. How Chemistry and Physics Meet in the Solid State. Angew. Chem. Int. Ed. 1987, 26, 846878. https://doi.org/10.1002/anie.198708461.

(18) Papoian, G.; Nørskov, J. K.; Hoffmann, R. A Comparative Theoretical Study of the Hydrogen, Methyl, and Ethyl Chemisorption on the $\mathrm{Pt}(111)$ Surface. J. Am. Chem. Soc. 2000, 122, 4129-4144. https://doi.org/10.1021/ja993483j.

(19) Varma, C. M.; Wilson, A. J. Systematics of the Binding Energy of Oxygen and Hydrogen on Transition-Metal Surfaces. I. Phys. Rev. B 1980, 22, 3795-3804. https://doi.org/10.1103/PhysRevB.22.3795.

(20) Hammer, B.; Norskov, J. K. Why Gold Is the Noblest of All the Metals. Nature 1995, 376, $238-240$. https://doi.org/10.1038/376238a0.

(21) Ruban, A.; Hammer, B.; Stoltze, P.; Skriver, H. L.; Nørskov, J. K. Surface Electronic Structure and Reactivity of Transition and Noble Metals. J. Mol. Catal. A Chem. 1997, 115, 421-429. https://doi.org/10.1016/S13811169(96)00348-2.

(22) Nørskov, J. K.; Abild-Pedersen, F.; Studt, F.; Bligaard, T. Density Functional Theory in Surface Chemistry and Catalysis. Proc. Natl. Acad. Sci. U. S. A. 2011, 108, 937-943. https://doi.org/10.1073/pnas.1006652108.

(23) Digne, M.; Sautet, P.; Raybaud, P.; Euzen, P.; Toulhoat, H. Use of DFT to Achieve a Rational Understanding of Acid-Basic Properties of Y-Alumina Surfaces. J. Catal. 2004, 226, 54-68. https://doi.org/10.1016/j.jcat.2004.04.020.

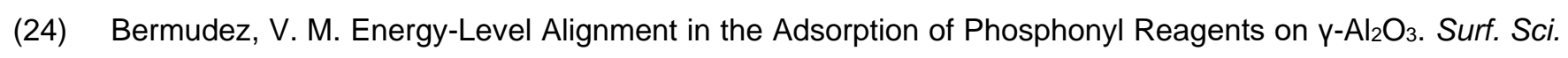
2008, 602, 1938-1947. https://doi.org/10.1016/j.susc.2008.03.036.

(25) Mowbray, D. J.; Martínez, J. I.; Calle-Vallejo, F.; Rossmeisl, J.; Thygesen, K. S.; Jacobsen, K. W.; Nørskov, J. K. Trends in Metal Oxide Stability for Nanorods, Nanotubes, and Surfaces. J. Phys. Chem. C 2011, 115, 2244-2252. https://doi.org/10.1021/jp110489u.

(26) Jenness, G. R.; Christiansen, M. A.; Caratzoulas, S.; Vlachos, D. G.; Gorte, R. J. Site-Dependent Lewis

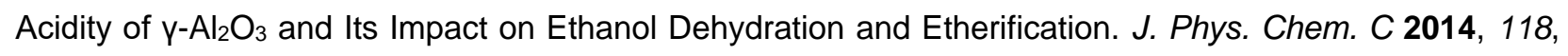
12899-12907. https://doi.org/10.1021/jp5028349.

(27) van Santen, R. A.; Tranca, I.; Hensen, E. J. M. Theory of Surface Chemistry and Reactivity of Reducible Oxides. Catal. Today 2015, 244, 63-84. https://doi.org/10.1016/j.cattod.2014.07.009.

(28) Kumar, G.; Lau, S. L. J.; Krcha, M. D.; Janik, M. J. Correlation of Methane Activation and Oxide Catalyst Reducibility and Its Implications for Oxidative Coupling. ACS Catal. 2016, 6, 1812-1821. https://doi.org/10.1021/acscatal.5b02657.

(29) Wang, P.; Fu, G.; Wan, H. How High Valence Transition Metal Spreads Its Activity over Nonmetal Oxoes: A Proof-of-Concept Study. ACS Catal. 2017, 7, 5544-5548. https://doi.org/10.1021/acscatal.7b01498.

(30) Castelli, I. E.; Man, I. C.; Soriga, S. G.; Parvulescu, V.; Halck, N. B.; Rossmeisl, J. Role of the Band Gap for the Interaction Energy of Coadsorbed Fragments. J. Phys. Chem. C 2017, 121, 18608-18614. https://doi.org/10.1021/acs.jpcc.7b04974.

(31) Hirunsit, P.; Toyao, T.; Siddiki, S. M. A. H.; Shimizu, K.; Ehara, M. Origin of $\mathrm{Nb}_{2} \mathrm{O}_{5}$ Lewis Acid Catalysis for 
Activation of Carboxylic Acids in the Presence of a Hard Base. ChemPhysChem 2018, 19, 2848-2857. https://doi.org/10.1002/cphc.201800723.

(32) Metiu, H.; Chrétien, S.; Hu, Z.; Li, B.; Sun, X. Chemistry of Lewis Acid-Base Pairs on Oxide Surfaces. J. Phys. Chem. C 2012, 116, 10439-10450. https://doi.org/10.1021/jp301341t.

(33) Helali, Z.; Jedidi, A.; Syzgantseva, O. A.; Calatayud, M.; Minot, C. Scaling Reducibility of Metal Oxides. Theor. Chem. Acc. 2017, 136, 100. https://doi.org/10.1007/s00214-017-2130-y.

(34) Capdevila-Cortada, M.; Vilé, G.; Teschner, D.; Pérez-Ramírez, J.; López, N. Reactivity Descriptors for Ceria in Catalysis. Appl. Catal. B Environ. 2016, 197, 299-312. https://doi.org/10.1016/j.apcatb.2016.02.035.

(35) Kamachi, T.; Tatsumi, T.; Toyao, T.; Hinuma, Y.; Maeno, Z.; Takakusagi, S.; Furukawa, S.; Takigawa, I.; Shimizu, K. Linear Correlations between Adsorption Energies and HOMO Levels for the Adsorption of Small Molecules on $\mathrm{TiO}_{2}$ Surfaces. J. Phys. Chem. C 2019, 123, 20988-20997. https://doi.org/10.1021/acs.jpcc.9b05707.

(36) Hirunsit, P.; Shimizu, K.; Fukuda, R.; Namuangruk, S.; Morikawa, Y.; Ehara, M. Cooperative $\mathrm{H}_{2}$ Activation at Ag Cluster $/ \theta-\mathrm{Al}_{2} \mathrm{O}_{3}(110)$ Dual Perimeter Sites: A Density Functional Theory Study. J. Phys. Chem. C 2014, 118, 7996-8006. https://doi.org/10.1021/jp5000792.

(37) Kovarik, L.; Bowden, M.; Shi, D.; Washton, N. M.; Andersen, A.; Hu, J. Z.; Lee, J.; Szanyi, J.; Kwak, J. H.; Peden, C. H. F. Unraveling the Origin of Structural Disorder in High Temperature Transition $\mathrm{Al}_{2} \mathrm{O}_{3}$ : Structure of $\theta-\mathrm{Al}_{2} \mathrm{O}_{3}$. Chem. Mater. 2015, 27, 7042-7049. https://doi.org/10.1021/acs.chemmater.5b02523.

(38) Murata, K.; Mahara, Y.; Ohyama, J.; Yamamoto, Y.; Arai, S.; Satsuma, A. The Metal-Support Interaction Concerning the Particle Size Effect of $\mathrm{Pd} / \mathrm{Al}_{2} \mathrm{O}_{3}$ on Methane Combustion. Angew. Chem. Int. Ed. 2017, 56, 15993-15997. https://doi.org/10.1002/anie.201709124.

(39) Liu, Y.; Li, Z. H.; Lu, J.; Fan, K.-N. Periodic Density Functional Theory Study of Propane Dehydrogenation over Perfect $\mathrm{Ga}_{2} \mathrm{O}_{3}(100)$ Surface. J. Phys. Chem. C 2008, 112, 20382-20392. https://doi.org/10.1021/jp807864z.

(40) Qu, J.; Zhou, X.; Xu, F.; Gong, X. Q.; Tsang, S. C. E. Shape Effect of Pd-Promoted $\mathrm{Ga}_{2} \mathrm{O}_{3} \mathrm{Nanocatalysts}$ for Methanol Synthesis by $\mathrm{CO}_{2}$ Hydrogenation. J. Phys. Chem. C 2014, 118, 24452-24466. https://doi.org/10.1021/jp5063379.

(41) Ye, J.; Liu, C.; Mei, D.; Ge, Q. Active Oxygen Vacancy Site for Methanol Synthesis from $\mathrm{CO}_{2} \mathrm{Hydrogenation}$ on $\ln _{2} \mathrm{O}_{3}(110)$ : A DFT Study. ACS Catal. 2013, 3, 1296-1306. https://doi.org/10.1021/cs400132a.

(42) Martin, O.; Martín, A. J.; Mondelli, C.; Mitchell, S.; Segawa, T. F.; Hauert, R.; Drouilly, C.; Curulla-Ferré, D.; Pérez-Ramírez, J. Indium Oxide as a Superior Catalyst for Methanol Synthesis by $\mathrm{CO}_{2}$ Hydrogenation. Angew. Chem. Int. Ed. 2016, 55, 6261-6265. https://doi.org/10.1002/anie.201600943.

(43) Albani, D.; Capdevila-Cortada, M.; Vilé, G.; Mitchell, S.; Martin, O.; López, N.; Pérez-Ramírez, J. Semihydrogenation of Acetylene on Indium Oxide: Proposed Single-Ensemble Catalysis. Angew. Chem. Int. Ed. 2017, 56, 10755 -10760. https://doi.org/10.1002/anie.201704999.

(44) Kresse, G.; Furthmüller, J. Efficient Iterative Schemes for Ab Initio Total-Energy Calculations Using a PlaneWave Basis Set. Phys. Rev. B 1996, 54, 11169-11186. https://doi.org/10.1103/PhysRevB.54.11169. 
(45) Kresse, G.; Furthmüller, J. Efficiency of Ab-Initio Total Energy Calculations for Metals and Semiconductors Using a Plane-Wave Basis Set. Comput. Mater. Sci. 1996, 6, 15-50. https://doi.org/10.1016/09270256(96)00008-0.

(46) Perdew, J. P.; Ruzsinszky, A.; Csonka, G. I.; Vydrov, O. A.; Scuseria, G. E.; Constantin, L. A.; Zhou, X.; Burke, K. Restoring the Density-Gradient Expansion for Exchange in Solids and Surfaces. Phys. Rev. Lett. 2008, 100, 136406. https://doi.org/10.1103/PhysRevLett.100.136406.

(47) Blöchl, P. E. Projector Augmented-Wave Method. Phys. Rev. B 1994, 50, 17953-17979. https://doi.org/10.1103/PhysRevB.50.17953.

(48) Grimme, S.; Ehrlich, S.; Goerigk, L. Effect of the Damping Function in Dispersion Corrected Density Functional Theory. J. Comput. Chem. 2011, 32, 1456-1465. https://doi.org/10.1002/jcc.21759.

(49) Gaussian 09; Gaussian, Inc., Wallingford, CT, 2009.

(50) Pedregosa, F.; Varoquaux, G. Scikit-Learn: Machine Learning in Python. J. Mach. Learn. Res 2011, 12, 2825-2830. https://doi.org/10.1007/s13398-014-0173-7.2.

(51) Geurts, P.; Ernst, D.; Wehenkel, L. Extremely Randomized Trees. Mach. Learn. 2006, 63, 3-42. https://doi.org/10.1007/s10994-006-6226-1.

(52) Toyao, T.; Suzuki, K.; Kikuchi, S.; Takakusagi, S.; Shimizu, K.; Takigawa, I. Toward Effective Utilization of Methane: Machine Learning Prediction of Adsorption Energies on Metal Alloys. J. Phys. Chem. C 2018, 122, 8315-8326. https://doi.org/10.1021/acs.jpcc.7b12670.

(53) Hinuma, Y.; Toyao, T.; Kamachi, T.; Maeno, Z.; Takakusagi, S.; Furukawa, S.; Takigawa, I.; Shimizu, K. Density Functional Theory Calculations of Oxygen Vacancy Formation and Subsequent Molecular Adsorption on Oxide Surfaces. J. Phys. Chem. C 2018, 122, 29435-29444. https://doi.org/10.1021/acs.jpcc.8b11279.

(54) Breiman, L.; Friedman, J.; Olshen, R.; Stone, C. Classification and Regression Trees; Wadsworth: Belmont, CA, 1984.

(55) Toyao, T.; Maeno, Z.; Takakusagi, S.; Kamachi, T.; Takigawa, I.; Shimizu, K. Machine Learning for Catalysis Informatics: Recent Applications and Prospects. ACS Catal. 2020, 10, 2260-2297. https://doi.org/10.1021/acscatal.9b04186.

(56) Bermudez, V. M. The Structure of Low-Index Surfaces of $\beta-\mathrm{Ga}_{2} \mathrm{O}_{3}$. Chem. Phys. 2006, 323, $193-203$. https://doi.org/10.1016/j.chemphys.2005.08.051.

(57) Pan, Y. X.; Liu, C. J.; Mei, D.; Ge, Q. Effects of Hydration and Oxygen Vacancy on $\mathrm{CO}_{2}$ Adsorption and

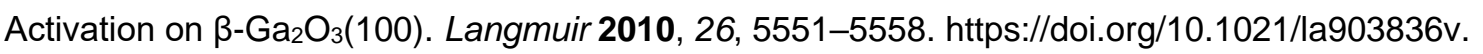

(58) Qu, J.; Tsang, S. C. E.; Gong, X. Q. A DFT Study on Surface Dependence of $\beta-\mathrm{Ga}_{2} \mathrm{O}_{3}$ for $\mathrm{CO}_{2} \mathrm{Hydrogenation}$ to $\mathrm{CH}_{3} \mathrm{OH}$. J. Mol. Model. 2014, 20, 2543. https://doi.org/10.1007/s00894-014-2543-7.

(59) Liu, T.; Feng, Z.; Li, Q.; Yang, J.; Li, C.; Dupuis, M. Role of Oxygen Vacancies on Oxygen Evolution Reaction Activity: $\quad \beta-\mathrm{Ga}_{2} \mathrm{O}_{3}$ as a Case Study. Chem. Mater. 2018, 30, 7714-7726. https://doi.org/10.1021/acs.chemmater.8b03015.

(60) Tanabe, K.; Hölderich, W. F. Industrial Application of Solid Acid-Base Catalysts. Appl. Catal. A Gen. 1999, 
181, 399-434. https://doi.org/10.1016/S0926-860X(98)00397-4.

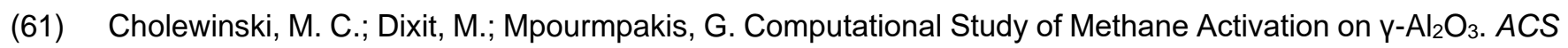
Omega 2018, 3, 18242-18250. https://doi.org/10.1021/acsomega.8b02554.

(62) Klimeš, J.; Michaelides, A. Perspective: Advances and Challenges in Treating van der Waals Dispersion Forces in Density Functional Theory. J. Chem. Phys. 2012, 137, 120901. https://doi.org/10.1063/1.4754130.

(63) Perdew, J. P.; Burke, K.; Ernzerhof, M. Generalized Gradient Approximation Made Simple. Phys. Rev. Lett. 1996, 77, 3865-3868. https://doi.org/10.1103/PhysRevLett.77.3865.

(64) Zhang, Y.; Yang, W. Comment on "Generalized Gradient Approximation Made Simple." Phys. Rev. Lett. 1998, 80, 890-890. https://doi.org/10.1103/PhysRevLett.80.890.

(65) Zhuang, H.; Tkalych, A. J.; Carter, E. A. Surface Energy as a Descriptor of Catalytic Activity. J. Phys. Chem. C 2016, 120, 23698-23706. https://doi.org/10.1021/acs.jpcc.6b09687.

(66) Tsuji, Y.; Yoshizawa, K. Adsorption and Activation of Methane on the (110) Surface of Rutile-Type Metal Dioxides. J. Phys. Chem. C 2018, 122, 15359-15381. https://doi.org/10.1021/acs.jpcc.8b03184.

(67) Takigawa, I.; Shimizu, K.; Tsuda, K.; Takakusagi, S. Machine-Learning Prediction of the d-Band Center for Metals and Bimetals. RSC Adv. 2016, 6, 52587-52595. https://doi.org/10.1039/c6ra04345c.

(68) Barone, P. M. V. B.; Camilo, A.; Galvão, D. S. Theoretical Approach to Identify Carcinogenic Activity of Polycyclic Aromatic Hydrocarbons. Phys. Rev. Lett. 1996, 77, 1186-1189. https://doi.org/10.1103/PhysRevLett.77.1186.

(69) Aptula, A. O.; Roberts, D. W.; Cronin, M. T. D. From Experiment to Theory: Molecular Orbital Parameters to Interpret the Skin Sensitization Potential of 5-Chloro-2-Methylisothiazol-3-One and 2-Methylisothiazol-3-One. Chem. Res. Toxicol. 2005, 18, 324-329. https://doi.org/10.1021/tx049715w.

(70) Vohs, J. M. Site Requirements for the Adsorption and Reaction of Oxygenates on Metal Oxide Surfaces. Chem. Rev. 2013, 113, 4136-4163. https://doi.org/10.1021/cr300328u. 
TOC

Molecular adsorption on group 13 metal oxide surfaces
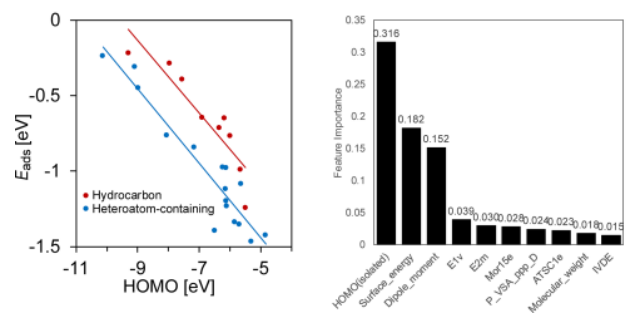

$(6.33 \mathrm{~cm} \times 4.75 \mathrm{~cm})$ 